\title{
Evaluation of decision-making in the treatment of acetabular fractures
}

\author{
Christof Audretsch ${ }^{1}{ }^{1}$, Alexander Trulson², Andreas Höch ${ }^{3}$, Steven C Herath ${ }^{1}$, Tina Histing ${ }^{1}$ and \\ Markus A Küper ${ }^{1}$ on behalf of Working Group on Pelvic Fractures of the \\ German Trauma Society
}

'BG Trauma Center, Department for Traumatology and Reconstructive Surgery, University of Tübingen, Tübingen, Germany

${ }^{2}$ BG Trauma Center, Department of Trauma Surgery, Murnau am Staffelsee, Germany

${ }^{3}$ Department of Orthopedics, Trauma and Plastic Surgery, University of Leipzig, Leipzig, Germany

Correspondence

should be addressed

to C Audretsch

Email

christofaudretsch@gmail.com
- Treatment of acetabular fractures is challenging and risky, especially when surgery is performed. Yet, stability and congruity of the hip joint need to be achieved to ensure early mobilization, painlessness, and good function. Therefore, coming up with an accurate decision, whether surgical treatment is indicated or not, is the key to successful therapy.

- Data from the German pelvic Trauma Registry $(n=4213)$ was evaluated retrospectively, especially regarding predictors for surgery. Furthermore, a logistic regression model with surgical treatment as the dependent variable was established.

- In total, $25.8 \%$ of all registered patients suffered from an acetabular fracture and $61.9 \%$ of them underwent surgery. The fracture classification is important for the indication of surgical therapy. Anterior wall fractures were treated surgically in $10.2 \%$, and posterior column plus posterior wall fractures were operated on in $90.2 \%$. Also, larger fracture gaps were treated surgically more often than fractures with smaller gaps (>3 mm 84.4\%, <1 $\mathrm{mm} 20 \%$ ). In total, $51.4 \%$ of women and $66.0 \%$ of men underwent surgery. Apart from the injury severity score (ISS), factors that characterize the overall picture of the injury were of no importance for the indication of a surgical therapy (isolated pelvic fracture: $62.0 \%$, polytrauma: $58.8 \%$ ). The most frequent reason for non-operative treatment was 'minimal displacement' in $42.2 \%$.

- Besides fracture classification and fracture characteristics, no factors characterizing the overall injury, except for the ISS, and unexpectedly gender, are important for making a treatment decision. Further studies are needed to determine the relevance of these factors, and whether they should be used for the decision-making process, in particular surgeons with less experience in pelvic surgery, can orient themselves to.

\author{
Keywords \\ - acetabular fracture \\ - gender medicine \\ - predictors for surgery \\ - epidemiology \\ $\checkmark$ pelvis \\ $\checkmark$ age
}

EFORT Open Reviews (2021) 7, 84-94

\section{Introduction}

Acetabular fractures are rare injuries with an incidence of $3 / 100,000 /$ year $(1,2)$. Therefore, therapy is often challenging, even for experienced trauma surgeons. The standard therapy used to be non-operative treatment for a long time (3). The groundbreaking work of Robert Judet and Emile Letournel in the mid-1960s led to a change in the decisions regarding the therapy regime $(3,4)$, and consequently, there has been an increase in the proportion of surgically treated fractures (1).

Aims of acetabular fracture management, either surgical or conservative, are in the short-term pain relief and early mobilization of the patients and in the long term, the prevention of posttraumatic osteoarthritis due to the incongruence of the hip joint.

The primary goal of surgery in acetabular fractures is the anatomic reconstruction of the articular surface to avoid long-term complications such as posttraumatic osteoarthritis with the need for arthroplasty. This is of great importance, especially in younger patients $(5,6)$. On the contrary, early treatment and mobilization should be aimed at the elderly in order to avoid secondary complications associated with immobilization $(7,8)$. This can often only 
be achieved satisfactorily by surgical treatment. Thus, in the short term, the aim of osteosynthetic treatment is the stabilization of the fragments and hence, a reduction in pain as well as the possibility of early mobilization. However, it must, of course, be ensured that even if surgical treatment is sought promptly (should it actually be necessary), this should al ways be carried out with the same quality and after equal careful preoperative preparation and improvement of the patient's overall status. Due to the demographic change, more elderly patients with an acetabular fracture were treated surgically $(1,2)$. However, older patients have a relatively higher perioperative mortality and morbidity risk (7) (e.g. from screw loosening due to lower bone quality) and additionally, they are at higher risk for a worse functional outcome of the injured hip joint $(5,6)$, whereas the functional requirements are lower.

In consequence, the decision for or against surgical treatment is often the key to successful treatment of acetabular fractures (9). For this purpose, various criteria have been described, additionally to the fracture classification, to support the decision process regarding both fracture-related and patient-related factors.

Fracture-related factors, which shift the treatment decision toward surgery, include the biomechanical stability (according to the fracture type based on the Letournel classification), the grade of displacement, and the grade of fracture comminution. For example, surgery is recommended for a roof-arc angle of $\angle 40^{\circ}$ in at least one radiographic view (anterior-posterior (AP) or $45^{\circ}$ oblique) of the pelvis $(9,10,11)$, as a parameter of fracture displacement. It should be noted, however, that many of these fracture-related factors date from a time when CT was not ubiquitous. A detailed CT assessment of the fracture is clearly the gold standard today. However, it is the aim of this work to evaluate under these exact circumstances which of the existing fracture-related factors are still used today for decision-making and thus are also applied in CT assessment. Furthermore, it is well known that the posterior wall is crucial for biomechanical joint stability. Posterior wall fractures, in general, and the grade of fracture comminution in posterior wall fractures in particular have been identified as a risk factor for a poor functional outcome (12), which in turn can result in a recommendation for surgical treatment of comminuted posterior wall fractures.

On the other hand, for patient-related factors, such as age, gender, and health status, data are still insufficient and multicenter studies are rare (13). This lack of scientific data is the reason why therapeutic decisions in acetabular fractures are still mainly based on the clinical experience of the surgeon. Since acetabular fractures are often treated in specialized centers, this lack of scientific data is often coped by the personal experience of the treating orthopedic surgeons. However, emergency diagnosis and treatment, as well as the initial decision for or against surgery and about the further course of therapy, including transfer to a specialized center, often have to be made in rural hospitals by surgeons with less experience in pelvic surgery. Factors that help to decide for or against surgical therapy might be of great help in those cases.

While some of the decision factors (especially fracturerelated factors) are well established, at least in the literature, it remains unknown (a) to what extent they have found their way into the decision-making process in everyday clinical practice and (b) to what extent other factors may have an influence and possibly even overpower these established decision factors.

Therefore, the aim of this retrospective registry study was (a) to specifically identify patient-related factors, which play a crucial role in making a decision regarding the treatment of acetabular fractures and (b) to compare their relevance and suitability to the established fracture-related decision factors. Finally, the criteria that were identified for the decision-making toward surgery were compared to the renowned reasons for non-operative therapy.

\section{Methods}

German Pelvic Registry

The German Pelvic Registry (GPR) is an international multicentric registry for patients with a pelvic fracture (14), with an increasing number of participating centers (currently 38) (15). The data are collected prospectively by the participating centers using a standardized questionnaire with 231 items. Personal data like age or gender, injury mechanism, injury extend according to the injury severity score (ISS)-system, and exact classification of the fractures according to the Tile-classification (for pelvic ring fractures), the Letournel classification (for acetabular fractures), and the Arbeitsgemeinschaft für Osteosynthesefragen/Orthopaedic Trauma Association (AO/OTA) classification (for pelvic ring and acetabular fractures) are part of the questionnaire as well as reasons for non-operative treatment. Furthermore, each pelvic ring or acetabular fracture is described exactly regarding the extent of displacement using radiography and/or CT. The GPR puts the focus on the surgical treatment of the respective fracture including operative and postoperative data. Besides the quality of reduction (measured by radiography and/or $\mathrm{CT}$ ), any postoperative complication is recorded until the discharge of the patient. Thanks to the large amount of datasets, the GPR allows to investigate questions regarding pelvic trauma management in rare conditions (16) as well as epidemiological questions (17).

The GPR was repeatedly approved by the Ethics Committee of the Chamber of Physicians of the Federal State of Saarland (No. 29/14). 


\section{E F O R T open revieus}

\section{Study population}

The database, which was used for this retrospective analysis, includes all datasets from 2003 (the start of the internetbased registry) to 2017 (before the questionnaire was updated). Data analysis was carried out in accordance with the approval of the local Ethics Committee of the EberhardKarls-University in Tübingen, Germany (430/2020BO).

\section{Patient selection}

From the total amount of 16359 cases, only cases with an acetabular fracture were included in this analysis (excluded $n=11$ 812). To obtain a patient population with only clearly assignable unilateral acetabular fractures, bilateral acetabular fractures were excluded (excluded $n=146$ ). Cases with a combination of a pelvic ring fracture and a unilateral acetabular fracture were included if the therapy for the acetabular fracture could be clearly assigned from the registry. Cases with ambiguous or unknown acetabular fracture classification were excluded (excluded $n=68$ ), as well as cases with missing values (excluded $n=120$ ). After this selection process, 4213 cases with a unilateral, classified acetabular fracture were included for further analysis (Fig. 1).

\section{Identification of fracture-related and patient-related factors}

For the patient-related factors, as well as for the fracturerelated factors, already well-described factors were examined, each with an unclear influence on the actual decision-making process. Furthermore, less-established factors that could potentially have a major influence on the decision-making process were examined as well.

Fracture types were classified according to Letournel and Judet (4). No surgical treatment is recommended when the remaining intact roof-arc angle is greater than $40^{\circ}$, the fracture step is smaller than $1 \mathrm{~mm}$, the fracture gap is smaller than $3 \mathrm{~mm}$, or ideally less than $1 \mathrm{~mm}$, and the comminution zone does not exceed $50 \%$, or is ideally not present at all $(5,9,10,11,12,18,19)$. According to that, the cut-off values were set within these variables. For the variable 'injury extend', the GPR database distinguishes between isolated pelvic fractures, multiple trauma (multiple injuries including the pelvis with an ISS $<16$ ), and polytrauma (multiple injuries including the pelvis with an ISS $\geq 16$ ). Fractures with additional relevant soft tissue damage or intrapelvic injuries, like vascular damage or open fractures, are graded as complex fractures. The transfer of patients from other smaller hospitals is often arranged with the intention of providing them with surgical therapy. Hence, the variable 'patient admission' distinguishes those patients from other patients who were admitted to the treating hospital primarily.

Differences in the share of surgical treatment of the categorical independent variables were evaluated with the chi-squared test for each fracture-related and patientrelated factors.

\section{Reasons for non-operative treatment}

The stated reasons for non-operative treatment were evaluated descriptively, and the percentage of their respective mentions was investigated, for the total of all cases but also broken down by age and gender.

\section{Regression model}

To assess the risk factors, the importance of various variables for the probability of surgical intervention was evaluated using odds ratios (OR) and their corresponding 95\% Cls.

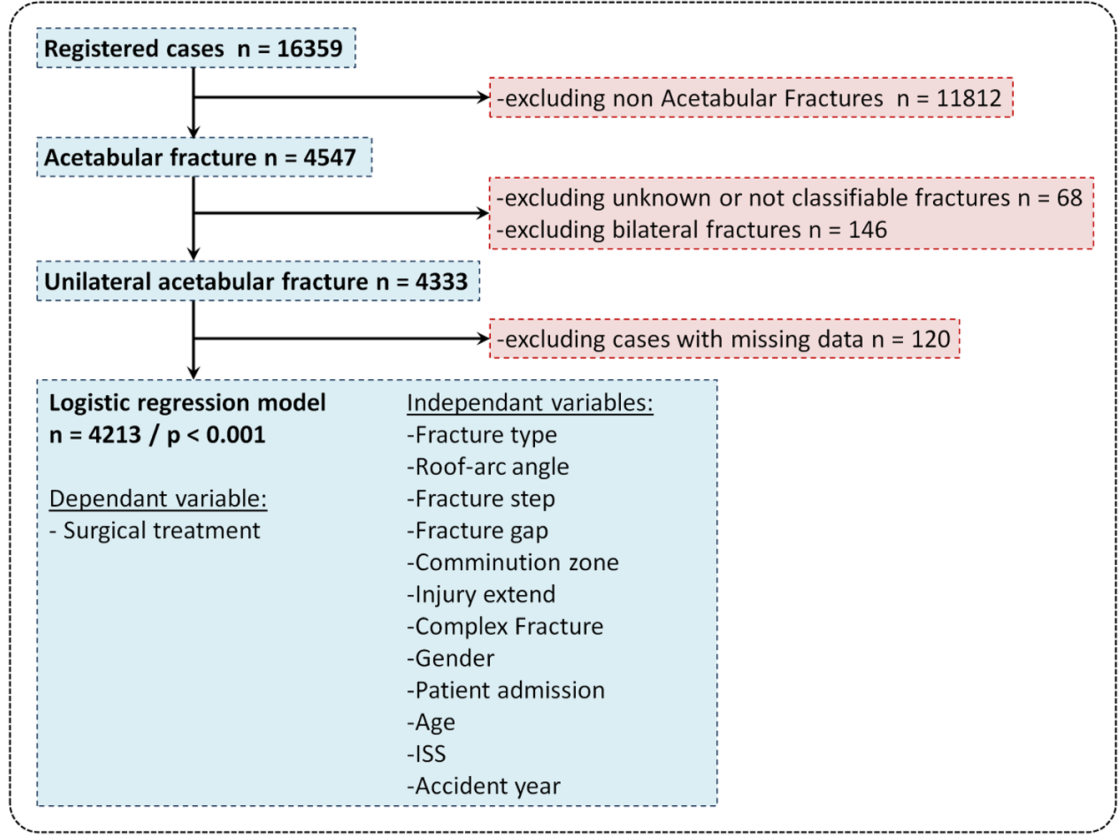

\section{Figure 1}

Patient selection and logistic regression model. This diagram shows the included and excluded cases as well as the variables of the logistic regression model. 
To identify confounders and to adjust the OR of these risk factors accordingly, a logistic regression model with the dependent, binomial variable 'surgical treatment' and the independent, categorical variables 'fracture type', 'roofarc angle', 'fracture step', 'fracture gap', 'comminution zone', 'injury extend', 'complex fracture', 'gender', and 'patient admission' as well as the independent, continuous variables 'age', 'ISS', and 'accident year' was established and subsequently applied. In order to rule out multicollinearity in the model, the variance inflation factor was determined additionally to the subjective evaluation of the correlation of the variables. No relevant evidence of multicollinearity could be found in the model tested.

The adjusted ORs as well as the risk factor-dependent probabilities for surgical treatment were evaluated with aid of a logistic regression model. In this model, the most common value, respectively the mean of the different variables, was used as reference. The probability for surgery at reference values is $0.75(95 \% \mathrm{Cl}: 0.70-0.80)$.

The level of significance is set at 5\% $(P=0.05)$. All analyses were completed using RStudio, Version 1.2 .5001 (20).

\section{Results}

\section{Patient-related factors as predictors for surgical treatment}

The mean age of the 4213 patients was $56.7 \pm 21.5$ years and showed an increasing trend over the years (2001-2009 mean $=52.3 \pm 20.9$ and $2010-2017$ mean $=60.0 \pm 21.3)$. In total, 3007 patients (71.4\%) were male and 1206 patients (28.6\%) were female. A total of 2606 patients (61.9\%) underwent surgery. Over the years, the percentage of patients treated surgically was constant (2001-2009, $62.3 \%$ and $2010-2017,61.4 \%)$.

In total, $66.2 \%(1986 / 3007)$ of the male patients underwent surgery, while $51.4 \%$ of women received surgical treatment (620/1206).

The mean ISS of the 4213 patients was $14.8 \pm 10.2$. Patients who underwent surgery for their acetabular fracture had a mean ISS of $14.3 \pm 9.3$, while patients with conservative therapy had a mean ISS of $15.6 \pm 11.4$ (Table $1)$.

In total, 2393 of the 4213 patients (56.8\%) were admitted primarily to the treating hospital, while 1820 of the 4213 patients (43.2\%) were transferred from another hospital, and $50.4 \%$ (1207/2393) of the primarily admitted patients underwent surgery, while $76.9 \%(1399 / 1820)$ of the transferred patients underwent surgery.

\section{Fracture-related factors as predictors for surgical treatment}

\section{Fracture type}

The three most common fracture types were anterior column fractures $(878 ; 20.8 \%)$, associated both column fractures $(722 ; 17.1 \%)$, and anterior column + posterior hemi transverse fractures $(699 ; 16.9 \%)$.

The fracture types with the highest rate of surgical treatment included combined posterior column + posterior wall fractures $(90.2 \%)$, transverse + posterior wall fractures (85.9\%), and associated both column fractures (85.2\%).

The fracture types with the lowest rate of surgical treatment included anterior wall fractures (10.2\%), anterior column fractures (42.2\%), and transverse fractures (50.5\%).

Grade of fracture displacement

With an AP view roof-arc angle of lesser than $40^{\circ}$, surgical therapy was chosen in the majority of the cases (69.2\%), whereas with an AP view roof-arc angle greater than $40^{\circ}$, the treatment strategy was slightly more often nonoperatively (51.7\%).

A fracture step bigger than $1 \mathrm{~mm}$ was a frequent indication for surgery (78.2\%), whereas a fracture with a step smaller than $1 \mathrm{~mm}$ was treated non-operatively in the majority of cases (65.6\%).

A fracture gap bigger than $3 \mathrm{~mm}$ was most often an indicator for an operative treatment (84.5\%), whereas a fracture gap smaller than $1 \mathrm{~mm}$ usually led to nonoperative treatment (80.1\%).

With a comminution zone present, surgical treatment was usually initiated (comminution zone $<51 \%$ : $79.1 \%$ surgically treated; $>50 \%: 86.7 \%$ ). Contrarily, without a comminution zone, non-operative treatment strategy was slightly predominant (53.3\%) (Table 2 ).

\section{Reasons for non-operative treatment}

For both men and women and across all patient age groups, the most frequently mentioned reasons for non-operative treatment were 'minimal displacement', followed by 'stable fracture'. 'General health' was the third

Table 1 Proportion and probability of surgical treatment of continuous variables. For each year of age and each ISS point, the probability for surgical treatment declines. The accident year has no influence.

\begin{tabular}{|c|c|c|c|c|c|c|c|}
\hline \multirow[b]{2}{*}{ Factor } & \multirow[b]{2}{*}{$\begin{array}{l}\text { Total, median } \\
\text { (mean } \pm \text { S.D.) }\end{array}$} & \multicolumn{2}{|c|}{ Surgery, median (mean \pm S.D.) } & \multicolumn{2}{|c|}{$\begin{array}{c}\text { Frequency of surgery at median } \\
\text { of total }\end{array}$} & \multirow[b]{2}{*}{$\begin{array}{l}\text { Adjusted OR } \\
(95 \% \mathrm{Cl})\end{array}$} & \multirow[b]{2}{*}{$\boldsymbol{P}$} \\
\hline & & Yes & No & $\begin{array}{l}\text { Surgery, Yes } \\
(n(\%))\end{array}$ & $\begin{array}{l}\text { Surgery, No } \\
(n(\%))\end{array}$ & & \\
\hline Age & $57(56.70 \pm 21.48)$ & $54(54.14 \pm 20.17)$ & $65(60.85 \pm 22.86)$ & $45(69.2)$ & $20(30.8)$ & $0.97(0.97-0.98)$ & $<0.001$ \\
\hline ISS & $9(14.80 \pm 10.17)$ & $9(14.28 \pm 9.31)$ & $9(15.63 \pm 11.38)$ & $1504(63.0)$ & $884(37.0)$ & $0.97(0.96-0.98)$ & $<0.001$ \\
\hline Accident year & $2010(2010.41 \pm 3.93)$ & $2010(2010.38 \pm 3.98)$ & $2010(2010.47 \pm 3.84)$ & $187(58.8)$ & $131(41.2)$ & $1.00(0.98-1.03)$ & 0.804 \\
\hline
\end{tabular}


most frequently mentioned reason for a decision against surgery (Table 3 ). However, the term 'general health' was not further specified.

\section{Regression model for factors that lead to surgical treatment Patient-related factors}

Factors that reduce the likelihood for surgical intervention were female gender (adjusted (adj.) OR: 0.79 (95\% Cl: $0.66-$ 0.95)), older age (adj. OR: 0.97 (95\% Cl: 0.97-0.98)) as well as an increasing ISS (adj. OR: 0.97 (95\% Cl, 0.96-0.98)).

Patients transferred from another hospital were more likely to undergo surgery (adj. OR: 1.90 (95\% Cl: 1.60 2.26)). However, other injury parameters, like 'polytrauma' (adj. OR: 1.06 (95\% Cl: 0.76-1.47)), 'multiple trauma' (adj. OR: 1.14 (95\% Cl: 0.92-1.42)), and 'complex pelvic trauma' (adj. OR: 1.30 (95\% Cl: 0.87-1.98)), as well as the year of the injury (adj. OR: 1.00 (95\% Cl: 0.98-1.03)), had no effect on the likelihood of undergoing surgical intervention (Fig. 2 and Table 4).

\section{Fracture type}

The logistic regression model shows an overall significance of $P<0.001$ and a misfit ratio of 0.185 , which would thus have correctly predicted the surgeon's decision in more than $81 \%$ of the cases. Fractures of the posterior wall (adj. OR: 2.09 (95\% Cl: 1.57-2.80)), especially in combination with a fracture of the posterior column (adj. OR: 5.58 (95\% Cl: 2.87-11.80)) or a transverse fracture (adj. OR: 3.70 (95\% Cl: 2.42-6.07)), increased the chance for a surgical intervention the most. Fractures of the anterior wall (adj. OR: 0.24 (95\% Cl: 0.16-0.36)) or anterior column (ref.) were less likely to be indicated for surgical treatment (Fig. 2 and Table 4).

\section{Grade of fracture displacement}

Further factors that influence the likelihood of surgical treatment independently were an intact AP roof-arc angle of $>40^{\circ}$ (adj. OR: 0.63 (95\% Cl: 0.52-0.75)), a small fracture gap of $<1 \mathrm{~mm}$ (adj. OR: 0.12 (95\% Cl: 0.09-0.15)) and a fracture step of $<1 \mathrm{~mm}$ (adj. OR: $0.55(95 \% \mathrm{Cl}$ : $0.45-$

Table 2 Non-operatively and surgically treated acetabular fractures. Characteristics of non-operatively and surgically treated acetabular fractures as well as the share of surgical treatment and the significance compared to the reference are shown. Data are presented as $n$ (\%).

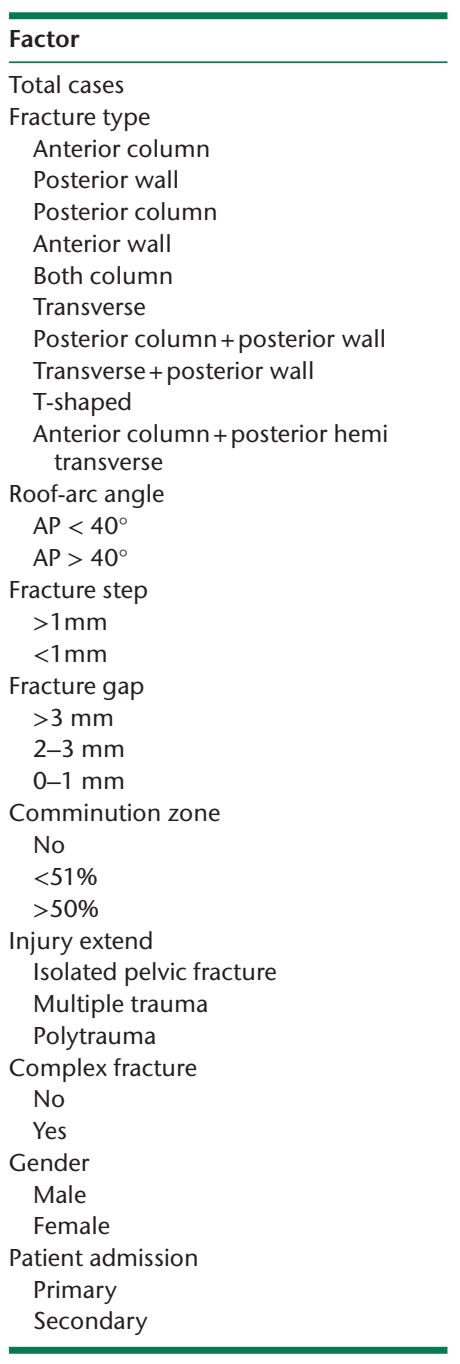

\begin{tabular}{|c|c|}
\hline Total & Surgical treatment \\
\hline 4213 & 2606 \\
\hline $878(20.8)$ & $371(14.2)$ \\
\hline $513(12.2)$ & 351 (13.5) \\
\hline $164(3.9)$ & $96(3.7)$ \\
\hline $374(8.9)$ & $38(1.5)$ \\
\hline $722(17.1)$ & 615 (23.6) \\
\hline $277(6.6)$ & $140(5.4)$ \\
\hline $122(2.9)$ & $110(4.2)$ \\
\hline $234(5.6)$ & $201(7.7)$ \\
\hline $230(5.5)$ & $160(6.1)$ \\
\hline $699(16.6)$ & $524(20.1)$ \\
\hline $2728(64.8)$ & $1889(72.5)$ \\
\hline $1485(35.2)$ & $717(27.5)$ \\
\hline $2640(62.7)$ & $2065(79.2)$ \\
\hline $1573(37.3)$ & $541(20.8)$ \\
\hline $2367(56.2)$ & 1999 (76.7) \\
\hline $813(19.3)$ & 401 (15.4) \\
\hline $1033(24.5)$ & $206(7.9)$ \\
\hline $2347(55.7)$ & 1095 (42.0) \\
\hline 1444 (34.3) & 1145 (43.9) \\
\hline $422(10.0)$ & $366(14.0)$ \\
\hline $2124(50.4)$ & $1316(50.5)$ \\
\hline $1097(26.0)$ & $707(27.1)$ \\
\hline $992(23.5)$ & $583(22.4)$ \\
\hline 3997 (94.9) & 2464 (94.6) \\
\hline $216(5.1)$ & $142(5.4)$ \\
\hline 3007 (71.4) & $1986(76.2)$ \\
\hline $1206(28.6)$ & $620(23.8)$ \\
\hline $2393(56.8)$ & $1207(46.3)$ \\
\hline $1820(43.2)$ & 1399 (53.7) \\
\hline
\end{tabular}

\begin{tabular}{c}
\hline Non-operative treatment \\
\hline 1607 \\
$507(31.5)$ \\
$162(10.1)$ \\
$68(4.2)$ \\
$336(20.9)$ \\
$107(6.7)$ \\
$137(8.5)$ \\
$12(0.7)$ \\
$33(2.1)$ \\
$70(4.4)$ \\
$175(10.9)$ \\
\\
$839(52.2)$ \\
$768(47.8)$ \\
$575(35.8)$ \\
$1032(64.2)$ \\
$368(22.9)$ \\
$412(25.6)$ \\
$827(51.5)$ \\
$1252(77.9)$ \\
$299(18.6)$ \\
$56(3.5)$ \\
$808(50.3)$ \\
$390(24.3)$ \\
$409(25.5)$ \\
$1533(95.4)$ \\
$74(4.6)$ \\
$1021(63.5)$ \\
$586(36.5)$ \\
$1186(73.8)$ \\
$421(26.2)$ \\
\end{tabular}

Surgical share $\%$ 61.9

$\boldsymbol{P}$ $\mathbf{P}$ Ref. $<0.001$ $<0.001$ $<0.001$ $<0.001$ 0.019 $<0.001$ $<0.001$ $<0.001$ $<0.001$ 75.0 $\begin{array}{cc}69.2 & \text { Ref. } \\ 48.3 & <0.001\end{array}$ 78.2 34.4 Ref. $<0.001$

\section{5} 49.3 19.9

Ref. $<0.001$ $<0.001$

\section{7}

79.3

86.7

Ref.

$<0.001$

$<0.001$

$62.0 \quad$ Ref.

$\begin{array}{ll}64.4 & 0.178\end{array}$

$\begin{array}{ll}58.8 & 0.097\end{array}$

61.6 Ref.

$\begin{array}{ll}65.7 & 0.257\end{array}$

66.0

51.4

Ref. $<0.001$

Ref.

$<0.001$ 


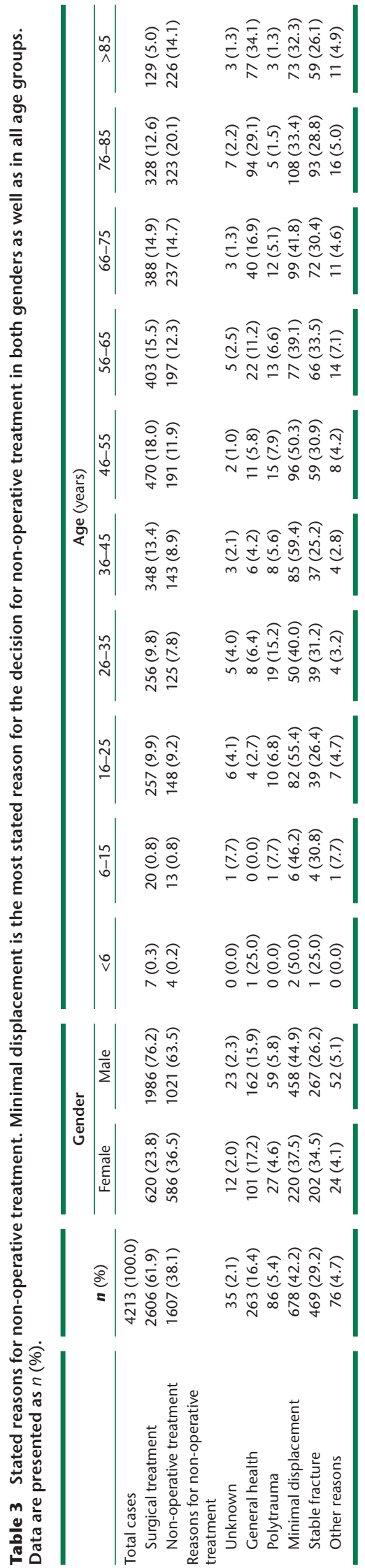

0.67)), as well as the extent of the comminution zone (adj. OR: 1.81 (95\% Cl: 1.29-2.59)) (Fig. 2 and Table 4).

\section{Discussion}

The question 'to operate or not to operate' is of crucial importance to orthopedic surgeons when planning the therapy of acetabular fractures. Factors, which contribute to the answer, are patient-related factors, fracture-related factors, surgeon-related factors, and hospital-related factors. Giannoudis et al. previously described different factors that influence the functional outcome after acetabular fractures (21). These factors can be divided into uncontrollable factors like age, fracture type, damage of femoral head, posterior displacement, associated injuries, and comorbidities and controllable factors, such as timing of surgery, surgical approach, local or systemic complications. Timely and, if possible, anatomic reconstruction of the acetabulum is considered particularly important in surgical therapy. This emphasizes the need for a stringent decision strategy from the beginning. Whenever surgical therapy is indicated, it should be performed timely, usually at a specialized center by surgeons experienced in acetabular surgery.

Regarding surgeon-related and hospital-related factors, one major decisive factor for or against an operation of acetabular fractures remains the experience of the trauma surgeon, as acetabular fractures are a relatively rare entity. In consequence, patients who need to undergo acetabular surgery are often transferred to specialized centers. However, though the major part of surgeries for acetabular fractures is performed in specialized trauma centers, surgeons in rural hospitals are often confronted with these patients and have to decide whether to transfer a patient with an acetabular fracture to another hospital for surgery or not. Moreover, regarding patient-related and fracturerelated factors, there is a lack of evidence, as prospectivecontrolled therapy studies are almost impossible in this field, even in a multicenter setting. Therefore, registry studies are essential to pool the experience of specialists in the treatment of acetabular fractures to help surgeons in rural hospitals, who, in most cases, treat patients with acetabular fracture first.

In this multicenter German Pelvic Registry (GPR) study, patients who were transferred to specialized centers underwent surgery significantly more often than patients who were admitted directly. The difference regarding the frequency of surgery between transferred and primarily admitted patients is obvious, since transfers are often initiated from smaller hospitals with the necessity of providing surgical care, while non-operative therapy can also be performed in rural hospitals, which usually are not participating in the GPR. Moreover, this shows that the rural hospitals already carry out a good selection of patients. 


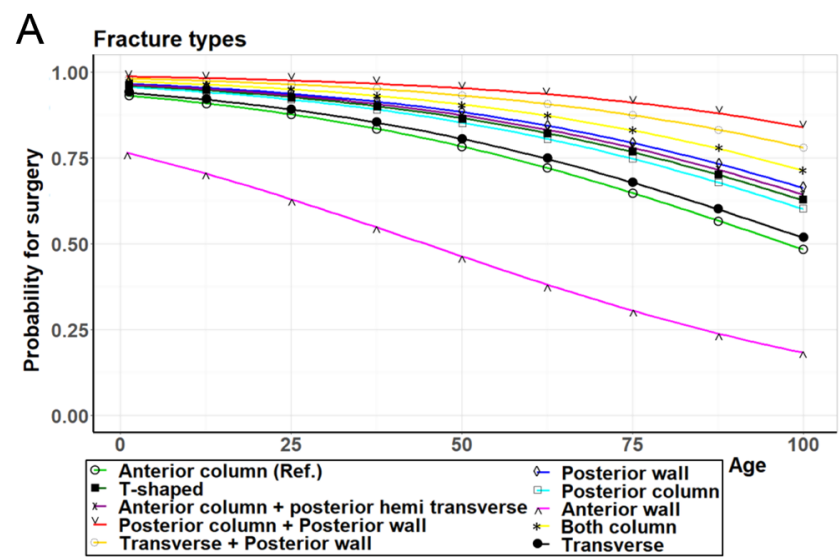

B
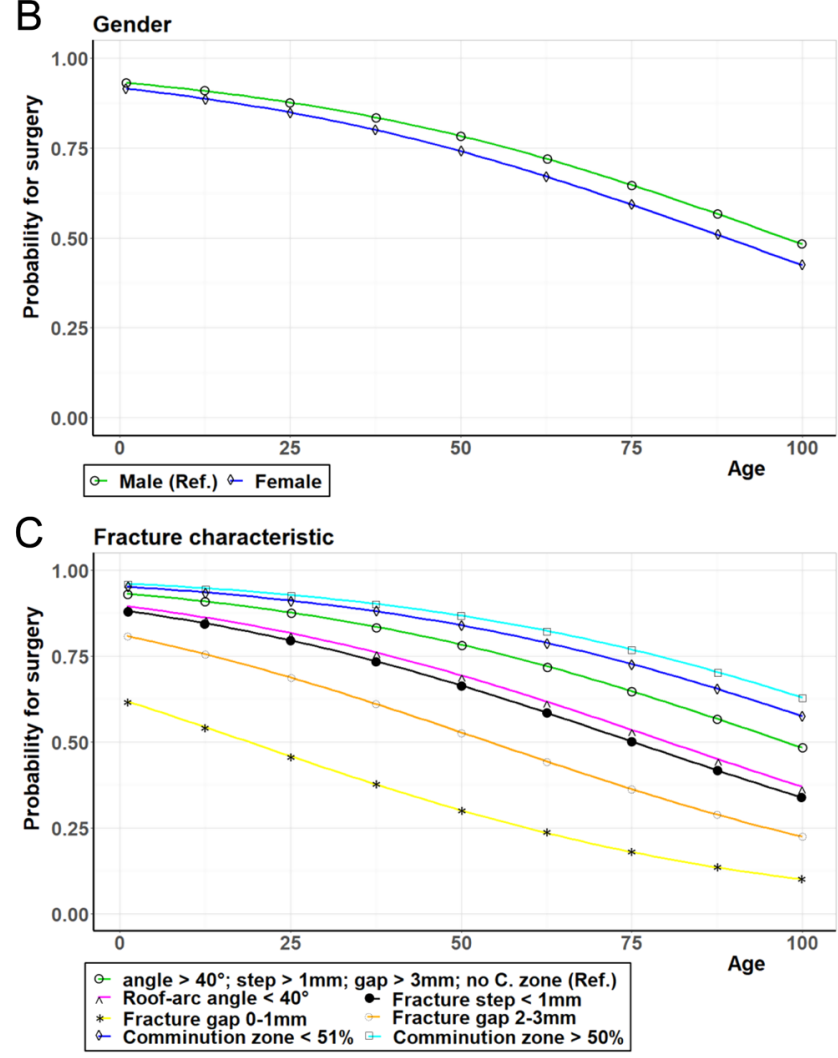

Figure 2

Probability for surgical intervention. The modeled probability for surgical intervention depending on the age is shown. (A) for the different fracture types; (B) for the genders, and (C) for different fracture characteristics.

\section{Patient-related factors}

With this study, we aimed to demonstrate which patientrelated factors play a role in the decision-making for experienced acetabular surgeons. However, due to the retrospective character of this analysis, it is not possible to evaluate whether the decision for or against surgery was based on these factors a priori. As expected, there are patient-related parameters, which appear to have a great influence on the decision-making. First, the patient's age significantly influences the treatment strategy. The main reasons for that can be found in some aspects of acetabular fractures in the elderly. It has been well discussed and examined in the past years that among elderly patients, the frequency of 'anterior' types of acetabular fractures increases $(1,2)$. This, combined with the fact that anterior fracture types are more unlikely to undergo surgery, one would have expected a decrease in the surgical procedures over the past years. However, we show that, despite increases in the mean age of the patients, the number of surgeries did not decrease. This might be explained by an overall upward tendency for surgical treatment that stands opposite to the age effect (1).

In our study, we were able to identify some other factors that are predictive criteria for the treatment strategy in acetabular fractures, which have not yet been described and discussed. Most surprisingly, female patients with acetabular fractures were less often treated surgically, though gender was not described as a factor for a different outcome (13). Possible confounding factors that were not queried in the registry and therefore cannot be part of the statistical model that is presented here could for example be the higher prevalence of osteoporosis among women, especially in older age $(22,23,24)$, and the dreaded complications of later or current pregnancies in younger patients $(25,26,27,28)$.

In cardiology and cardio-surgery, gender is known as an independent, often inexplicable factor, for the choice of treatment strategy and outcome. The amount of transfused blood products, like PRBC for example, differs significantly between men and women despite matched initial conditions (29). Beyond that, even the fact that, whether the diagnosis and therapy are carried out by a male or female physician is reported to be of crucial importance for the survival rates of women, following acute myocardial infarctions (30). Future studies might therefore need to verify this gender effect, explain it, and evaluate its influence and possible confounders in a targeted and ideal prospective manner.

\section{Fracture-related factors}

The most common acetabular fracture is the anterior column fracture with an AP roof-arc angle $<40^{\circ}$, a fracture step $>1 \mathrm{~mm}$, a fracture gap $>3 \mathrm{~mm}$, and without comminution zone.

Of course, there are differences between operatively and non-operatively treated acetabular fractures. The fracture type, however, is still one of the most decisive predictors for surgical treatment, with the Letournel/Judet classification still being the most important classification system. However, particularly factors that characterize the fracture and especially its grade of displacement more detailed, like the AP roof-arc angle, fracture gap, fracture step, 
Table 4 Crude and adjusted odds ratio (OR) of surgical treatment. The crude as well as the adjusted odds ratio is shown for the categorical values used in the analysis.

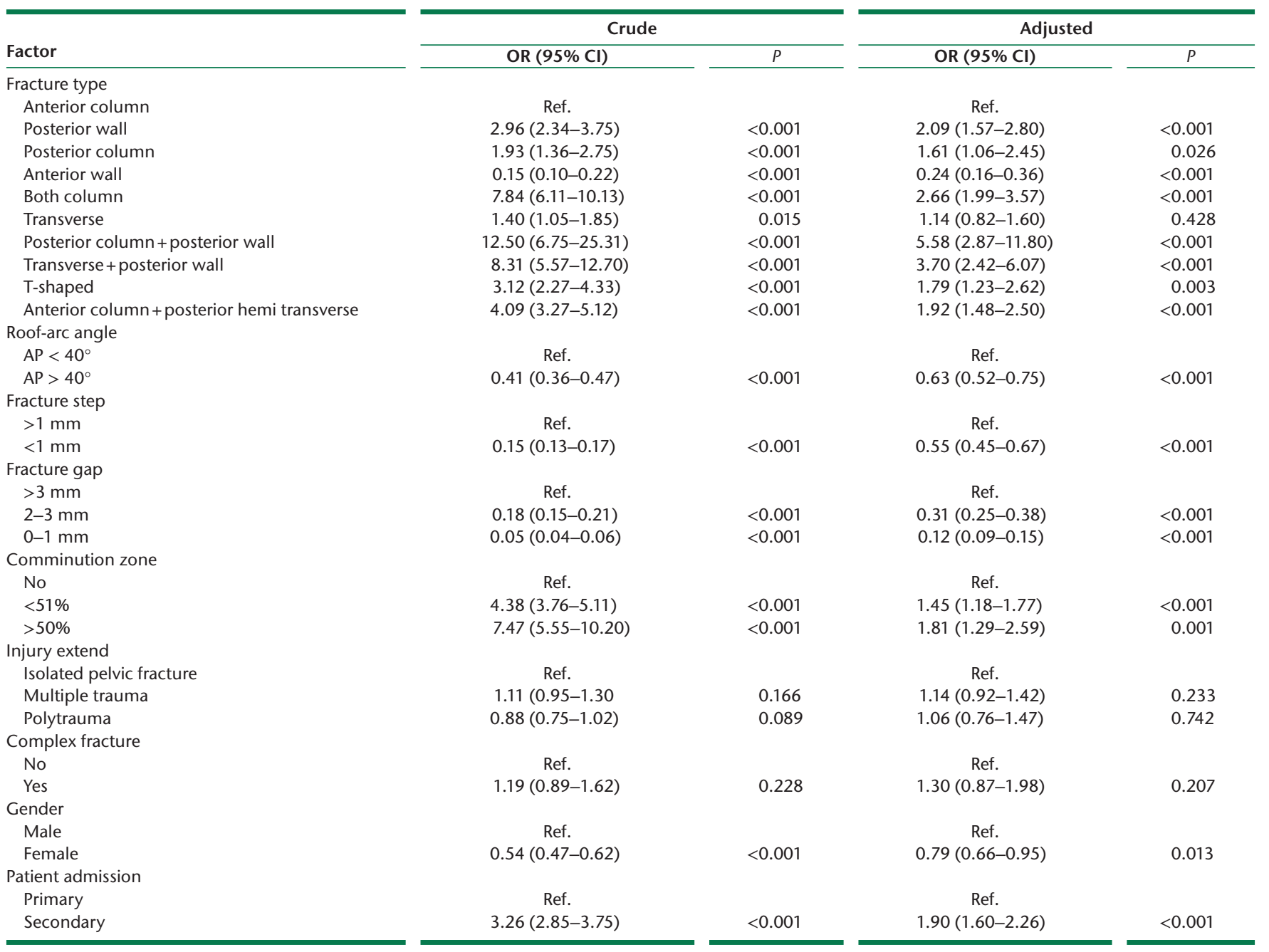

and comminution zone, are of high importance, which is represented in the updated AO/OTA classification system (31). These factors are also well described in the literature $(5,9,10,11,12,18,19)$ and, as shown previously, seem to be well accepted and have become part of the routine decision-making of specialized acetabular surgeons.

The registry data show that there is clearly a lower probability for surgical treatment if a fracture involves the anterior acetabular column - especially the anterior wall - compared to a fracture of the posterior column - especially the posterior wall. The biomechanically plausible rationale, which has already been well described in the literature, is the fact that fractures of the posterior wall are often unstable luxation fractures and that the posterior wall is exposed to a higher load and is therefore more important for the functionality and long-term outcome than the anterior wall $(5,6,32,33)$.

Except for the ISS, factors that describe the injury in general, such as isolated pelvic fractures vs multiple trauma or polytrauma, or association of the fracture with additional pelvic soft tissue injuries (which are by definition complex pelvic injuries) seem to be irrelevant for the decision-making process. However, complex pelvic fractures with an incidence of only $5.1 \%$ among all acetabular fractures are relatively rare; this should be considered in the assessment of the effect and its significance. It has already been shown that combined injuries, for example, of the pelvis and abdomen, play an important role in the surgical course and have an influence on complications and mortality of patients with pelvic fractures (34).

\section{Reasons for non-operative therapy}

Most frequently, only minimal fracture displacement was stated as one of the reasons for non-operative therapy, followed by a stable fracture. While minimal displacement can be accurately defined by the fracture gap and fracture step, the classification of a stable 
fracture, however, requires a complex consideration depending on the fracture type and especially on the experience of the surgeon. Fracture-related parameters as well as the patient's constitution and demands, especially regarding deterioration of the variables 'fracture gap' and 'fracture step', must be considered. This is also reflected by the great influence that these two factors have on the therapy decision within our model. '(Poor) general health' as a reason for non-operative therapy is probably best reflected by age and ISS. Other factors that describe the overall condition of the patient and the fracture itself had no influence on our model (Tables 1, 2 and 3). Moreover, particularly older patients have an increased perioperative risk for complications, due to the, usually, very invasive surgical procedures. However, this is offset by the significantly increased risks from immobilization during conservative therapy, particularly in older patients $(5,6,7,8)$. This is why White et al., for example, state that old age should not be a reason to decide against surgical treatment (35).

\section{Study limitations and context}

As the GPR only provides data for the time of in-hospital treatment, it cannot be concluded whether the fracturerelated or patient-related factors have an impact on the outcome. Therefore, a definition of new cut-off values for those factors cannot be made based on the findings in this study. To answer this question, the respective patientrelated factors that were found in the present study should be ideally evaluated in separate prospective studies based on the GPR.

Some of the factors, especially the fracture-related factors like the Letournel classification or the grade of displacement, are well accepted in their importance to help decide whether to perform surgery. Usually, these factors can be seen as strong decision-making factors, as there is already strong evidence regarding a positive outcome. On the other hand, for patient-related factors like age, gender, extent of injury, or co-morbidities, there is less evidence regarding a positive outcome, though those data are usually given as demographic data in most of the outcome studies. Especially patient-related factors like underaged patients or critically injured patients might be a source of misinterpretation of the results. We decided against excluding certain patient groups a priori for two reasons: First, as many factors as possible should be included as decision triggers in the logistic regression model. Secondly, in consequence, the exclusion of specific groups might distort the results, especially as randomly set limits like age or injury severity should have no influence on the decision-making process. Whether they do influence the decision-making, is exactly the target of interest of this work.
Another limitation of the retrospective study design is the difficulty to distinguish between the graduated impact of the respective factors on the decision-making. However, by choosing a logistic regression model, the influence of the different variables on the decision-making process can be estimated. However, there is a risk of multicollinearity if several factors influence the decision in the same way. To rule out multicollinearity as a possible source of misinterpretation of the results, the 'variance inflation factor' was determined and multicollinearity could be ruled out in the tested model. Nevertheless, in a model with many different variables, the individual variables will always influence each other, and must ultimately be computationally separated from each other, as was done in this study.

Influenced by the work of Robert Judet and Emile Letournel in the mid-1960s, there has been a significant shift over the last century from conservative therapy to surgical treatment $(1,3,4,35)$. Thus, the indications for surgical and conservative therapy were also subject to change over time. Especially displaced fractures as well as fractures of the quadrilateral surface and fractures of biomechanically important structures, such as the posterior wall, often led to unsatisfactory results in the conservative therapeutic approach (35).

Finally, the importance of surgical treatment of acetabular fractures in order to achieve a good bone stock for delayed hip arthroplasty is controversially discussed and has not yet been accepted as a decisive factor for the therapy strategy $(35,36,37)$.

\section{Importance of this study}

The aim of this registry study was to identify positive predictors for acetabular surgery from a large, multicenter pelvic trauma registry, where a distinct expert level for the treatment of acetabular fractures is available. These predictors might be of great help in the future for trauma surgeons with less experience in pelvic trauma surgery to correctly plan the therapy of patients with acetabular fractures. The good reproducibility of the decisions by the logistic regression model ( $80 \%$ correct predictions) shows that based on the factors presented here, decisions were largely made uniformly by the experts. At the same time, the model can also serve as a helpful tool in decisionmaking, yet it obviously cannot cover all eventualities (20\% incorrect predictions). Thus, the decision about the correct treatment strategy remains the responsibility of the attending physician and should always be made with a lot of professional expertise, taking into account patient-related, fracture-related, surgeon-related, and hospital-related factors. This work offers guidance in this decision-making process, as it presents the current state of expert knowledge regarding the suitability of the 
above-mentioned decision factors, in combination with an evaluation of those factors on which a decision is made most often.

\section{Conclusion}

Besides fracture classification, and factors that characterize the fracture itself, age and gender influence the decision whether surgery is performed or not. Surgery is performed less frequently in females and with increasing age, but the tendency for surgical treatment has not changed over the years despite the demographic development. Except for the ISS, factors that characterize the total image of the injury are of no importance. It seems that factors other than those usually discussed play a role in the decision-making process regarding an operative or non-operative therapy strategy. Future studies are needed to determine the relevance of these factors and whether they should be taken into consideration. However, in any case, it is advisable to be aware of the criteria on which the decision is based.

ICMJE Conflict of Interest Statement

M A K has received travel grants from BG Unfallklinik Tübingen. The authors declare that there is no conflict of interest that could be perceived as prejudicing the impartiality of this study.

\section{Funding Statement}

This work did not receive any specific grant from any funding agency in the public, commercial, or not-for-profit sector.

\section{Author contribution statement}

$\mathrm{C} \mathrm{A}$ and $\mathrm{M} \mathrm{A} \mathrm{K}$ designed the study. C A acquired the data from the German Pelvic Registry. C A and M A K did the data analysis. C A prepared and wrote the article. All authors interpreted the data, revised the manuscript regarding scientific input, and approved the submitted final version.

\section{Acknowledgement}

The authors thank Mrs Catharina Scheuermann-Poley for editing this manuscript in the English language.

\section{References}

1. Ochs BG, Marintschev I, Hoyer H, Rolauffs B, Culemann U, Pohlemann T \& Stuby FM. Changes in the treatment of acetabular fractures over 15 years: analysis of 1266 cases treated by the German Pelvic Multicentre Study Group (DAO/DGU). Injury 201041 839-851. (https://doi.org/10.1016/j.injury.2010.04.010)

2. Mears DC. Surgical treatment of acetabular fractures in elderly patients with osteoporotic bone. Journal of the American Academy of Orthopaedic Surgeons 19997 128-141. (https:// doi.org/10.5435/00124635-199903000-00006)

3. Prevezas N. Evolution of pelvic and acetabular surgery from ancient to modern times. Injury 200738 397-409. (https://doi.org/10.1016/j.injury.2007.01.035)

4. Letournel E \& Judet R. Fractures of the Acetabulum. Ed RA Elson. Berlin Heidelberg: Springer, 1993. (https://doi.org/10.1007/978-3-642-75435-7)
5. Verbeek D0, List JP van der, Tissue CM \& Helfet DL. Predictors for long-term hip survivorship following acetabular fracture surgery: importance of gap compared with step displacement. Journal of Bone and Joint Surgery: American Volume 2018100 922-929. (https://doi.org/10.2106/JBJS.17.00692)

6. Tannast M, Najibi S \& Matta JM. Two to twenty-year survivorship of the hip in 810 patients with operatively treated acetabular fractures. Journal of Bone and Joint Surgery: American Volume 201294 1559-1567. (https://doi.org/10.2106/JBJS.K.00444)

7. Vallier HA, Cureton BA, Ekstein C, Oldenburg FP \& Wilber JH. Early definitive stabilization of unstable pelvis and acetabulum fractures reduces morbidity. Journal of Trauma 201069 677-684. (https://doi.org/10.1097/TA.0b013e3181e50914)

8. Firoozabadi R, Cross WW, Krieg JC \& Routt MLC. Acetabular fractures in the senior population-epidemiology, mortality and treatments. Archives of Bone and Joint Surgery 20175 96-102. (https://doi.org/10.22038/abjs.2016.7933)

9. Grubor P, Krupic F, Biscevic $\mathbf{M}$ \& Grubor $\mathbf{M}$. Controversies in treatment of acetabular fracture. Medical Archives 201569 16-20. (https://doi.org/10.5455/ medarh.2015.69.16-20)

10. Matta JM \& Merritt PO. Displaced acetabular fractures. Clinical Orthopaedics and Related Research 1988230 83-97. (https://doi.org/10.1097/00003086-198805000-00009)

11. Matta JM, Anderson LM, Epstein HC \& Hendricks P. Fractures of the acetabulum. A retrospective analysis. Clinical Orthopaedics and Related Research 1986205 230-240. (https://doi.org/10.1097/00003086-198604000-00029)

12. Kim HY, Yang DS, Park CK \& Choy WS. Modified Stoppa approach for surgical treatment of acetabular fracture. Clinics in Orthopedic Surgery 20157 29. (https://doi. org/10.4055/cios.2015.7.1.29)

13. Murphy D, Kaliszer M, Rice J \& McElwain JP. Outcome after acetabular fracture. Prognostic factors and their inter-relationships. Injury $2003 \mathbf{3 4}$ 512-517. (https://doi. org/10.1016/s0020-1383(02)00349-2)

14. Pohlemann T, Tosounidis $G$, Bircher M, Giannoudis P \& Culemann U. The German Multicentre Pelvis Registry: a template for an European expert network? Injury 2007 38 416-423. (https://doi.org/10.1016/j.injury.2007.01.007)

15. Holstein JH, Stuby FM, Herath SC, Culemann U, Aghayev E \& Pohlemann T. Influence of the pelvic trauma registry of the DGU on treatment of pelvic ring fractures. Unfallchirurg 2016119 475-481. (https://doi.org/10.1007/s00113-016-0168-2)

16. Audretsch CK, Mader D, Bahrs C, Trulson A, Höch A, Herath SC, Küper MA \& Working Group on Pelvic Fractures of the German Trauma Society. Comparison of pelvic C-clamp and pelvic binder for emergency stabilization and bleeding control in type-C pelvic ring fractures. Scientific Reports 202111 2338. (https://doi.org/10.1038/s41598-021-81745-z)

17. Höch A, Pieroh P, Gras F, Hohmann T, Märdian S, Holmenschlager F, Keil H, Palm HG, Herath SC, Josten C, et al. Age and 'general health' - beside fracture classification - affect the therapeutic decision for geriatric pelvic ring fractures: a German Pelvic Injury Register Study. International Orthopaedics 201943 2629-2636. (https://doi. org/10.1007/s00264-019-04326-w)

18. Kim HT, Ahn J-M, Hur J-0, Lee J-S \& Cheon S-J. Reconstruction of acetabular posterior wall fractures. Clinics in Orthopedic Surgery 20113 114-120. (https://doi. org/10.4055/(ios.2011.3.2.114)

19. Matta JM. Fractures of the acetabulum: accuracy of reduction and clinical results in patients managed operatively within three weeks after injury. Journal of Bone and Joint Surgery 1996 78-A 1632-1645. (https://doi.org/10.1055/s-0030-1267077)

20. Team R. RStudio: Integrated Development Environment for $R ;$ v 1.2.5001. Boston, MA, USA: RStudio, Inc., 2019. 


\section{E F O R T open revieus}

21. Giannoudis PV, Grotz MRW, Papakostidis C \& Dinopoulos H. Operative treatment of displaced fractures of the acetabulum. A meta-analysis. Journal of Bone and Joint Surgery: British Volume 200587 2-9. (https://doi.org/10.1302/0301-620X.87B1.15605)

22. Breuil V, Roux CH, Testa J, Albert C, Chassang M, Brocq 0 \& EullerZiegler L. Outcome of osteoporotic pelvic fractures: an underestimated severity. Survey of 60 cases. Joint Bone Spine 200875 585-588. (https://doi.org/10.1016/j.jbspin.2008.01.024)

23. Johnell 0 \& Kanis J. Epidemiology of osteoporotic fractures. Osteoporosis International 200516 (Supplement 2) S3-S7. (https://doi.org/10.1007/s00198-004-1702-6)

24. Anderson PA, Froyshteter AB \& Tontz WL. Meta-analysis of vertebral augmentation compared with conservative treatment for osteoporotic spinal fractures. Journal of Bone and Mineral Research 201328 372-382. (https://doi.org/10.1002/ jbmr.1762)

25. Amorosa LF, Amorosa JH, Wellman DS, Lorich DG \& Helfet DL. Management of pelvic injuries in pregnancy. Orthopedic Clinics of North America 201344 301-315, viii. (https://doi.org/10.1016/j.ocl.2013.03.005)

26. Leggon RE, Wood GC \& Indeck MC. Pelvic fractures in pregnancy: factors influencing maternal and fetal outcomes. Journal of Trauma 200253 796-804. (https:// doi.org/10.1097/00005373-200210000-00033)

27. Cannada LK \& Barr J. Pelvic fractures in women of childbearing age. Clinical Orthopaedics and Related Research 2010468 1781-1789. (https://doi.org/10.1007/ s11999-010-1289-5)

28. Flik K, Kloen P, Toro JB, Urmey W, Nijhuis JG \& Helfet DL. Orthopaedic trauma in the pregnant patient. Journal of the American Academy of Orthopaedic Surgeons 200614 175-182. (https://doi.org/10.5435/00124635-200603000-00008)

29. Shevde K, Pagala M, Kashikar A, Tyagaraj C, Shahbaz N, Iqbal M \& Idupuganti R. Gender is an essential determinant of blood transfusion in patients undergoing coronary artery bypass graft procedure. Journal of Clinical Anesthesia 200012 109-116. (https://doi.org/10.1016/s0952-8180(00)00120-3) 